\title{
EXPERIENCIA CLINICA EN EL MANEJO DE FRACTURAS DE PENE EN EL HOSPITAL UNIVERSITARIO DEL VALLE (CALI-COLOMBIA)
}

\author{
Jaime Alejandro Restrepo, Carlos Gonzalo Estrada, Herney Andres García y Jorge Carbonell.
}

Servicio de Urología. Hospital Universitario del Valle. Universidad del Valle. Cali. Colombia.

\begin{abstract}
Resumen.- OBJETIVO: El objetivo principal del presente estudio fue describir las características clínicas de los pacientes con diagnóstico de fractura de pene en el Hospital Universitario del Valle (Cali, Colombia).

MÉTODOS: Se realizó un estudio descriptivo, en el que se revisaron todas las historias clínicas de los pacientes con diagnóstico de fractura de pene entre enero de 2001 a diciembre de 2008 en el Hospital Universitario del Valle (HUV) en la ciudad de Cali. Se tuvieron en cuenta variables relacionadas con los antecedentes urológicos, la etiología, el diagnóstico de la fractura, el procedimiento quirúrgico y el seguimiento. Se realizó un análisis univariado con el programa estadístico STATA v. 10.1
\end{abstract}

RESULTADOS: Se encontraron 18 casos de fractura de pene con un promedio de edad de 30 años. 11 pacientes $(61 \%)$ presentaron el episodio secundario a la relación sexual. Se presentó edema, dolor y chasquido más frecuentemente. El diagnóstico fue clínico en el $100 \%$ de los casos. El abordaje quirúrgico se realizó con una incisión circunferencial en la mayoría de los pacientes. El cuerpo cavernoso derecho fue el más frecuentemente lesionado y en la mayoría de los casos la lesión fue corregida con una sutura absorbible de forma continua. Los pacientes tuvieron una hospitalización posquirúrgica en promedio de 1.5 días.

CONCLUSIONES: La fractura de pene es una entidad cuyo diagnóstico es clínico, debe realizarse de forma precoz y la reparación quirúrgica es el tratamiento de elección para evitar complicaciones y permitir que el paciente retorne a su actividad sexual satisfactoria de manera temprana.

Palabras clave: Fractura de pene. Cuerpos cavernosos. Uretra. Pene.

Summary.- OBJECTIVES: The main objective of this study was to describe the clinical characteristics of patients diagnosed with penile fracture in the Hospital Universitario del Valle (Cali, Colombia).

Herney Andrés García

Cr 35 No $3^{a}-38$ Ap 301. Cali (Colombia).

andresgarcia125@yahoo.com

Aceptado para publicar: 20 de junio 2009
METHODS: A descriptive study, reviewing all the medical records of patientsdiagnosed with penile fracture from January 2001 to December 2008 at Hospital Universitario del Valle (HUV) in Cali. It took into account variables related to urological history, etiology, diagnosis, the surgical treatment and followup. Univariate analysis was performed with the statistical program STATA v. 10.1 
RESULTS: There were 18 cases of penile fracture with an average age of 30 years. 11 patients $(61 \%)$ had episodes related to intercourse. Patients presented swelling, pain and popping or cracking sound. The diagnosis was done by history and physical examination in 100\%. Surgery was performed with a subcoronal incision in most of the patients. The right corpus cavernosum was frequently injured and corrected with absorbable suture. The patients had an average of 1.5 days of postoperative hospital stay.

CONCLUSIONS: Penile fracture is an entity the diagnosis of which is straightforward and can be reliable by history and physical examination. Surgical repair is the treatment of choice preventing complications, allowing the patient to return to satisfactory sexual life.

Keywords: Penile fracture. Corpora cavernosa. Urethra. Penis.

\section{INTRODUCCIÓN}

La fractura de pene (llamada erróneamente así a pesar de que el hombre no cuente con hueso peneano a diferencia de otros mamíferos) o fractura de cuerpos cavernosos es una urgencia urológica poco frecuente, sin embargo dentro de los traumatismos genitales es el más comúnmente descrito (1).

La fractura de pene se describe como la ruptura de la túnica albugínea de los cuerpos cavernosos debido a un traumatismo cerrado (2). Es difícil conocer la verdadera incidencia de esta patología ya que existen muchos casos no publicados y probablemente muchos pacientes no consultan por tratarse de una situación embarazosa (3). Existen diferentes mecanismos de trauma peneano que varían en frecuencia según el área geográfica, ocupando el primer lugar en EEUU el acto sexual, en el Mediterráneo la manipulación genital no asociada a deseo sexual, en Japón la masturbación y en los países musulmanes las maniobras para forzar la detumescencia (4).

La presentación clínica es típica: los pacientes reportan un chasquido acompañado de dolor, detumescencia y posteriormente edema y deformidad (5). El diagnóstico es clínico y el tratamiento más aceptado es el quirúrgico precoz, que consiste en la exposición y rafia de los cuerpos cavernosos (6).

El objetivo principal del presente estudio fue describir las características clínicas de los pacientes con diagnóstico de fractura de pene en el Hospital Universitario del Valle (Cali, Colombia).

\section{MATERIALES Y MÉTODOS}

Se realizó un estudio descriptivo transversal. Se revisaron todas las historias clínicas de los pacientes masculinos mayores de 14 años con diagnóstico CIE 10 de fractura de pene entre enero de 2001 a diciembre de 2008 que llegaron al servicio de Urología del Hospital Universitario del Valle (HUV) en la ciudad de Cali.

Se tuvieron en cuenta variables como: edad, fecha de consulta, antecedente de patología genital, tiempo entre el ingreso y la cirugía y el mecanismo de la fractura (Relación sexual, masturbación, otras). Con respecto al diagnóstico se tuvieron en cuenta características como dolor, chasquido, detumescencia, edema, hematoma, solución de continuidad en el cuerpo del pene, uretrorragia, retención urinaria, si el diagnóstico fue hecho por hallazgos clínicos o ecografía, si se realizó uretrografía previa.

Se evaluó en la exploración quirúrgica el tipo de incisión, la presencia de hematoma, la uni o bilateralidad de la lesión de cuerpos cavernosos, el compromiso uretral, el lado de la lesión, la localización (distal, medio o proximal), el tipo de sutura que se utilizó (absorbible, no absorbible), el calibre de la sutura, el tipo de cierre del cuerpo cavernoso (continua, discontinua), el tiempo posquirúrgico de hospitalización.

A pesar de no haber podido seguir a todos los pacientes, se identificaron variables como la presencia de curvatura del pene o de dolor con la erección.

\section{Análisis estadístico}

Se realizó un análisis univariado con tablas de frecuencia, medidas de tendencia central y dispersión y gráficos acordes al tipo de variable. El análisis se realizó en el programa estadístico STATA v. 10.1 Se siguieron las indicaciones de la resolución 8430 de 1993 y la declaración de Helsinki (Tokio 2004) con respecto a investigación en humanos. Se trata de una investigación sin riesgo (A).

\section{RESULTADOS}

Se encontraron 17 pacientes ( 18 casos dado que 1 paciente presentó en dos ocasiones fractura de pene) entre enero de 2001 y diciembre de 2008.

La edad promedio fue $30+-8.1$ años (Figura 1). Fue más frecuente encontrar pacientes durante los años 2006 y 2008 (Tabla I). 


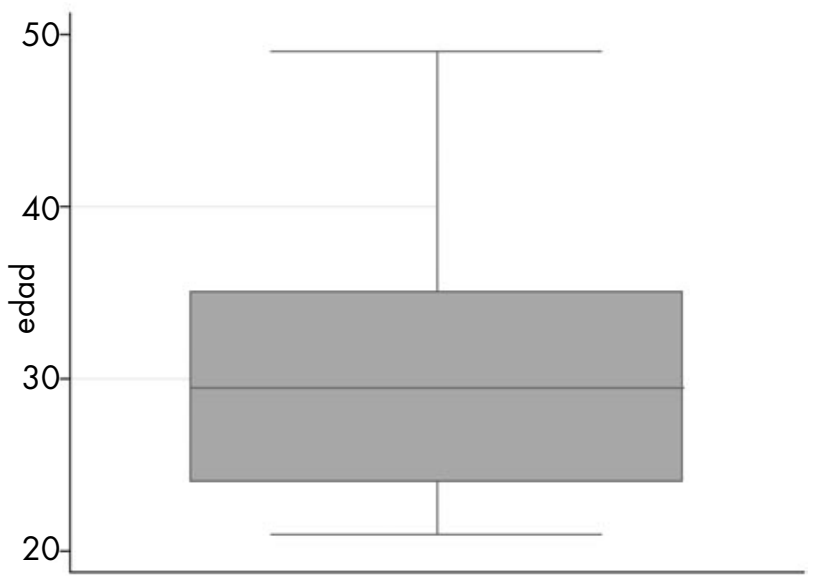

FIGURA 1. Distribución por edad de los casos de fractura de pene en el Hospital Universitario del Valle entre enero de 2001 a diciembre de 2008.

No se encontraron pacientes con antecedentes urológicos como enfermedad de peyronie, angulación congénita del pene u otra patología genital. Sin embargo como ya previamente se describió, 1 paciente presentó en dos ocasiones fractura de pene (una en el año 2001 y otra en el 2006)

El tiempo entre la consulta al servicio de urgencias y la realización del procedimiento quirúrgico presentó una mediana de 19 horas (mín 6.5 Máx 87).

\section{TABLA I. FRECUENCIA DE PRESENTACIÓN DE CASOS POR CADA AÑO ENTRE ENERO DE 2001 A DICIEMBRE DE 2008 EN EL HOSPITAL UNIVERSITARIO DEL VALLE.}

\begin{tabular}{|c|c|c|}
\hline AÑO & FRECUENCIA & PORCENTAJE \\
\hline 2001 & 1 & 5 \\
\hline 2002 & 3 & 16 \\
\hline 2003 & 2 & 11 \\
\hline 2004 & 3 & 16 \\
\hline 2005 & 0 & 0 \\
\hline 2006 & 4 & 22 \\
\hline 2007 & 1 & 5 \\
\hline 2008 & 4 & 22 \\
\hline
\end{tabular}

Once pacientes $(61.1 \%)$ acusan a la relación sexual como el principal desencadenante de los síntomas y 7 pacientes $(38.8 \%)$ tuvieron otro ( 4 pacientes se manipularon el pene en erección durante la micción, 1 paciente tuvo el episodio dormido, 1 paciente tuvo una torsión voluntaria del pene, 1 paciente tuvo una caída a horcajadas). No se presentaron episodios con masturbación.

La presentación clínica de los pacientes más frecuente fue con edema, dolor y chasquido (Tabla II). El diagnóstico fue clínico en el $100 \%$ de los casos y a ningún paciente se le realizó uretrografía previo al procedimiento quirúrgico.

Con respecto al procedimiento quirúrgico: $\mathrm{Se}$ realizó una incisión circunferencial en 10 pacientes $(55 \%)$ y lateral en 8 casos (44.4\%). Se encontró hematoma a la exploración en el $100 \%$ de los casos.

Hubo lesión de un cuerpo cavernoso en 16 casos (88.8\%) y bilateral en 2 pacientes (11.1\%). En aquellos casos que se encontró lesión bilateral también se evidenció lesión uretral. 8 pacientes (44.4\%) tuvieron lesión del cuerpo cavernoso derecho, 7 pacientes $(38.8 \%)$ del izquierdo y en 3 pacientes no se logró encontrar el dato.

Con respecto a la localización de la lesión: 1 paciente $(5.5 \%)$ tuvo lesión distal, 5 pacientes

\section{TABLA II. PRESENTACIÓN CLÍNICA DE LOS PACIENTES CON FRACTURA DE PENE DE ENERO 2001 A DICIEMBRE DE 2008 EN EL HOSPITAL UNIVERSITARIO DEL VALLE.}

\begin{tabular}{|c|c|c|}
\hline CARACTERISTICA & FRECUENCIA & PORCENTAJE \\
\hline DOLOR & 12 & 66.6 \\
\hline CHASQUIDO & 11 & 61.1 \\
\hline DETUMESCENCIA & 5 & 27.7 \\
\hline EDEMA & 15 & 83.3 \\
\hline HEMATOMA & 8 & 44.4 \\
\hline URETRORRAGIA & 1 & 5 \\
\hline RETENCIÓN URINARIA & 0 & 0 \\
\hline SOLUCIÓN DE CONTINUIDAD & 0 & 0 \\
\hline
\end{tabular}


(27.7\%) tuvieron lesión en tercio medio y 10 pacientes $(55.5 \%)$ tuvieron lesión en el tercio proximal. En 2 pacientes no se encontró el dato.

En todos los casos se realizó el cierre con sutura de tipo absorbible. En 10 pacientes (55.5\%) fue de calibre 3.0 y en 8 pacientes $(44.4 \%)$ fue de calibre 4.0. En 17 casos (94.4\%) el cierre fue de forma continua. Un paciente tuvo el cierre con puntos separados.

Los pacientes tuvieron una hospitalización posquirúrgica en promedio de $1.5+0.78$ días. 10 pacientes $(55.5 \%)$ se hospitalizaron 1 día, 7 pacientes $(38.8 \%) 2$ días y 1 paciente $(5.5 \%)$ duró 4 días hospitalizado.

Se realizó seguimiento a 14 casos, 4 (28.5\%) presentaron dolor con la erección y 1 paciente $(7.1 \%)$ presentó curvatura de pene.

\section{DISCUSIÓN}

La fractura de pene es una entidad poco frecuente, en la mayor serie de casos publicada en población iraní se presentó 1 caso semanal (7); se desconoce la incidencia real dada la variabilidad de las situaciones que lo originan y a lo embarazoso que resulta a los pacientes consultar.

Su etiología es el trauma cerrado lo que usualmente requiere que el pene esté en erección (8).

Es secundario generalmente a una curvatura exagerada y súbita del pene en erección, con posterior ruptura de uno o los dos cuerpos cavernosos que puede estar acompañado de lesión uretral entre 0 $-38 \%$ de los casos $(2,9)$.

Los mecanismos de trauma de las fracturas de pene en nuestro centro coinciden con los descritos en los diferentes artículos, siendo los principales la relación sexual $(4,10,11)$, las maniobras para la detumescencia o manipulación forzada del pene, así como darse la vuelta en la cama con el pene erecto, manipulación peneana nocturna inconsciente, caída con pene en erección o trauma directo en el pene $(3,9,12)$.

La sintomatología que referían los pacientes al ingreso a la unidad de urgencias del Hospital Universitario del Valle no era diferente a los síntomas clásicos de la fractura de pene: audición de un chasquido acompañado por edema, dolor y detumescencia del pene con formación de hematoma, dando el aspecto de una berenjena $(1,6,13,14)$.
El diagnóstico de la fractura de pene es clínico, basado en una adecuada historia clínica y un examen físico exhaustivo, aunque si se presenta duda se pueden utilizar otras estrategias como la ecografía doppler de pene que muestra la existencia de un hematoma por debajo de la fascia de Buck, así como la disrupción de la túnica albugínea (15). Otros estudios son la cavernosografía que tiene un alto porcentaje de falsos negativos (15\%) y presenta complicaciones como alergia, fibrosis e infección 11 y la resonancia magnética nuclear ( $R M N$ ) que es costosa y no está disponible en la mayoría de los servicios de urgencias (9). Nuestros hallazgos son acordes con lo descrito previamente en la literatura pues en ninguno de los casos se requirió de ayudas diagnósticas previas al procedimiento quirúrgico. La uretrografía es un estudio invasivo que sólo debe realizarse ante la sospecha de trauma uretral, sin embargo es un estudio que puede tener hasta $14 \%$ de falsos negativos; en nuestro centro, ningún paciente fue sometido previamente a este estudio, por el contrario, durante el procedimiento quirúrgico fue evaluada la uretra mediante el paso de una sonda y la visualización directa del sitio de la lesión.

Aunque existe el manejo conservador de las fracturas de pene, ésta fue utilizada hasta los años 60 's como estándar de oro; se describió principalmente para fracturas pequeñas sin compromiso uretral, sin embargo presenta complicaciones entre 10 - 80\% (Erección dolorosa, masas pulsátiles, sobreinfección del hematoma, erecciones parciales) (16-18). El tratamiento de elección para esta entidad desde los años 70's es la exploración quirúrgica y rafia primaria del defecto $(6,9,13,14)$. En nuestra institución todos los pacientes con sospecha de fractura de pene fueron llevados a exploración quirúrgica, donde el tipo de incisión (subcoronal o lateral) dependió del sitio de fractura donde se sospechaba que estuviese el defecto en los cuerpos cavernosos. Existen diferentes abordajes (infrapúbico, inguinoescrotal y peneano), sin embargo el más utilizado es el peneano y dentro de éste existen dos abordajes: el subcoronal circular y el longitudinal. El más utilizado y aceptado es el subcoronal, aunque no hay diferencias en el uso de los dos tipos (9).

Usualmente la ruptura es de un cuerpo cavernoso, más frecuentemente en el tercio proximal $(91 \%)$, y el cierre debe realizarse con sutura de tipo absorbible calibre 3/0 a 4/09. En nuestro estudio no hubo predominio marcado por lesión en alguno de los lados, mostrando una ligera tendencia hacia el lado derecho, así como el segmento que se comprometió en la mayoría de las ocasiones fue el proximal, coincidiendo con lo publicado en la literatura (9). 
La lesión uretral puede estar presente hasta en $10 \%-38 \%$ de los casos (9). En este estudio todos los pacientes que presentaron lesión bilateral de cuerpos cavernosos concomitaron con lesión uretral $(11 \%)$, manifestando así la magnitud del trauma que originó la fractura de pene, aunque de éstos sólo se encontró uretrorragia en un paciente.

Aunque el seguimiento de los pacientes no fue completo ( 14 casos) y fue corto, mostró una baja incidencia de curvatura de pene y un porcentaje no despreciable de erecciones dolorosas, que es acorde con la literatura mundial.

Es claro que los estudios de tipo descriptivos son los más susceptibles a presentar diferentes tipos de sesgos, sin embargo nuestro trabajo muestra la experiencia de 8 años del Hospital Universitario del Valle, un centro de referencia en la ciudad de Cali donde se atiende la población sin seguridad social del suroccidente colombiano y esta información nos sirve como abordaje exploratorio para generar hipótesis para nuevos estudios, dada la baja incidencia de esta entidad.

\section{CONCLUSIÓN}

La fractura de pene es una entidad cuyo diagnóstico es clínico, debe realizarse de forma temprana y la reparación quirúrgica es el tratamiento de elección para evitar complicaciones y permitir que el paciente retorne a su actividad sexual satisfactoria de manera precoz.

\section{BIBLIOGRAFIA y LECTURAS RECOMENDADAS (*lectura de interés $y^{* *}$ lectura fundamental)}

1. Hunter W, Layron L. Penile and Genital Injuries. Urol Clin N Am 2006; 33: 117-126.

2. Gil P, Borque A, Valdivia P, Rodríguez L, Rioja L. Fractura de pene: aportación de un caso. Actas Urol Esp 2000; 24 (3): 272-274.

3. Fergany AF, Angermeier KW, Montague DK. Review of Cleveland clinic experience with penile fracture. Urology 1999; 54: 352-355.

4. Jack G, Garraway I, Reznichek R, Rajfer J. Current Treatment Options for Penile Fractures. Rev Urol 2004; 6(3):114-120.
5. Koifman L, Cavalcanti A, Manes C, Filho D, Favorito L. Penile fracture - Experience in 56 cases. Int Braz J Urol 2003; 29: 35-9.

**6. Tejido A, Martin M, Villacampa F, De la Morena J, Suarez J, Leiva O. Tratamiento quirúrgico de la fractura de pene. Nuestra experiencia. Actas Urol Esp 1999; 23 (9): 784-788.

**7. Zargooshi J. Penile fracture in Kermanshah, Irán: report of 172 cases. J Urol., 164: 364, 2000

*8. Mariño J, Senovilla J, Mateos J, Abengozar A, Sevilla M, Caballero $\mathrm{M}$ et al. Fractura de pene. Revisión de la literatura y presentación de dos casos. Actas Urol Esp 2000; 24 (9): 767-770.

**9. Serrano A, Golbano J, Gonzalez-peramato P, Otero I, Sancho J, Arroyo J. et al. Fractura de pene: evaluación diagnóstica y actitudes terapéuticas. Revisión de la literatura. Arch Esp Urol 2001; 54(8): 803-810.

*10. Rubio J, Sanchez F, Segura A, Regalado R, Caffaratti J, Ruiz-Castañé E et al. Tratamiento quirúrgico de la fractura de cuerpos cavernosos y uretra: revisión de la literatura a propósito de dos casos. Arch. Esp. Urol. 1996; 49(5): 499-506.

11. Anselmo G, Fandella A, Faggiano L. Fractures of the penis: therapeutic approach and longterm results. Br. J. Urol. 1991; 67: 509.

12. Asgari MA, Hosseini SY, Safarinejad MR. Samadzadeh B., Bardideh AR. Penile fractures: evaluation, therapeutic approaches and long-term results J Urol 1996; 155: 148-149.

*13. Lema J, Blanco M, Cimadevilla A, Rodríguez H, Tato J, Cimadevilla Covelo A. Fractura de pene con afectación de cuerpos cavernosos y uretra. Actas Urol. Esp. 1999; 23(10): 900-903.

*14. Martínez J, Pastor H, Carrión P, Giménez JM, Donate MJ, Virseda JA. Fractura de cuerpos cavernosos. Serie de casos. Actas Urol Esp. 2008; 32(6):599-602

*15. Catala L, Rapariz MA, Valero J, Belon JA, Isorna S, Aleman P. Fractura de pene: valor del ecodoppler-color. Arch. Esp. Urol.1998; 51(8): 831834

16. Meares EM. Traumatic rupture of the corpus cavernosum. J Urol 1971; 105: 407

17. Nicolaisen GS, Melamud A, Williams RD. McAninch JW. Rupture of the corpus cavernosum: surgical management J Urol 1983; 130: 917-919.

18. Kalash S, Young Jr J. Fracture of penis: controversy of surgical versus conservative treatment. Urology 1984; 24: 21-24. 